\title{
Herramientas financieras del mercado venezolano como alternativas de inversión
}

\author{
Atencio Cárdenas, Edith* \\ González, Alejandro** \\ González, Blanca*** \\ Katiuska Marín****
}

\begin{abstract}
Resumen
La dinámica natural y los procesos de cambio de los países que intervienen en el concierto económico-financiero actual, hacen que resulte cada vez más necesario el análisis de la situación Financiera de la empresa, siguiendo tanto su propio desarrollo interno como su posición frente al resto de las empresas que intervienen en el mismo mercado. Por lo tanto el objetivo del presente trabajo es determinar cuales de las herramientas de inversión son adecuadas para aplicar en la empresa, logrando acertar la mejor política de inversión. El tipo de estudio es descriptivo, para lo cual se realizó una revisión documental de Modigliani y Millar quienes fueron los primeros estudiosos de los métodos y aproximaciones para la toma de decisiones financieras y sobre los modos de financiación de las empresas como instrumento técnico, desarrollados en la década de los años 50 hasta la actualidad. Los resultados demuestran que las alternativas de inversión siguen teniendo el mismo patrón decisorio, sujeto a alternativas diversas todo dentro del contexto contemporáneo en concordancia con las nuevas tendencias de inversión del mercado. A manera de conclusión se pone de manifiesto una cierta acepción del valor de la empresa ligada a la valoración del accionista y las obligaciones que se tienen. Sin embargo se logra resaltar como el análisis financiero asume otra noción de valor cuando se refiere a la posición que adoptan los eventuales vendedores y compradores de una empresa o parte más o menos importante de la misma, considerando a las herramientas financieras del mercado venezolano como excelentes opciones para la realización de inversiones acorde con el capital que disponga la empresa.
\end{abstract}

Palabras clave: Herramienta financiera, sistemas económicos, empresa, inversión y mercado.

Recibido: 08-02-08. Aceptado: 20-07-08

* $\quad$ Lic. en Administración, Magíster en Gerencia de Empresas, cursante del Doctorado en Administración de Empresas UPM-LUZ. Profesora e Investigadora de la Universidad José Gregorio Hernández (UJGH). E-mail: edithsoraida@yahoo.es

** Lic. en Contaduría Pública. Magíster en Gerencia de Empresa. Profesor e Investigador de UJGH.

*** Lic en Administración. Magíster en Gerencia de Empresas. Cursante del Doctorado en Ciencias Sociales. Profesor e investigador de la UJGH.

**** Lic. en Administración. Cursante de la Maestría en Gerencia Pública. LUZ.

E-mail: katymarin@yahoo.es 
Herramientas financieras del mercado venezolano como alternativas de inversión Atencio Cárdenas, Edith; González, Alejandro; González, Blanca y Katiuska Marín

\title{
Financial Tools of the Venezuelan Market as Investment Alternatives
}

\begin{abstract}
The natural dynamic and the processes of change in countries participating in the current economic-financial concert, make it ever more necessary to analyze the financial situation of the company, following its own internal development as well as its position in the face of the rest of the companies that participate in the same market. Therefore, the objective of this study is to determine which investment tools are adequate for application to the company, achieving thereby, the best investment policy. The type of study is descriptive, performing a documentary review of Modigliani and Millar, who were the first to study the methods and approaches for financial decision making and financing modes for companies as a technical instrument, developed in the decade from the 50 s to the present. Results show that investment alternatives continue to have the same decision-making pattern, subject to diverse alternatives all within the contemporary context in concordance with new market investment tendencies. By way of conclusion, a certain meaning for the value of the company is manifested linked to a valuation of the stockholders and the obligations they have. Nevertheless, the study is able to emphasize how financial analysis assumes another notion of value when it refers to the position adopted by eventual buyers and sellers of a company or a more or less important part of the same, considering the financial tools of the Venezuelan market as excellent options for making investments according to the capital that the company has at its disposal.
\end{abstract}

Key words: Financial tool, economic systems, company, investment, market.

\section{Introducción}

El contexto económico se caracteriza por una sucesión de situaciones, en las cuales los hechos y fenómenos que afectan a la empresa actual, en todos sus niveles, tienen lugar con una rapidez y profundidad sin precedentes en la historia. El objetivo de este artículo es determinar cuales de las herramientas de inversión son adecuadas para aplicar en la empresa, para lo cual se utilizo la metodología basada en el análisis crítico descriptivo. Es por ello que se plantea que los entes económicos, cuyo objetivo consiste en la prestación de servicios o cesión de productos financieros, realizan sus actividades en un marco que resulta cada vez más complejo, pero esta complejidad queda, además, acentuada por la gran concurrencia existente en este sector, como consecuencia de la continua y "aparentemente" variada oferta de productos y servicios financieros que se pueden hallar en el mercado cuya característica "real" es su elevada homogeneidad. Estos hechos hacen necesario, cuando no imprescindible, la creación de una reserva o banco de ideas, destinada a mantener un stock de productos aptos para ser lanzados en el mercado cuando éste lo precise. En este sentido, el esfuerzo que se ven obligados a realizar los ejecutivos de las empresas para la adaptación de nuevas situaciones es importante, pero a su vez es indispensable si se desea conseguir la supervivencia en esta época de extrema y casi brutal competen- 
cia. No solo se debe tomar en cuenta lo anterior, sino que ellos tendrán en sus manos el tomar decisiones financieras de inversión, subsistema prioritario en un sistema financiero empresarial, el cual debe ser manejado con inteligencia $y$ sumo cuidado para que las alternativas de inversión seleccionadas sean las mas acertadas y convenientes para la empresa. Sin embargo estas empresas en el ejercicio de su actividad financiera, se ven afectadas por un elevado número de interacciones que proceden tanto del propio ámbito económico como del entorno social, cultural, político, entre otros. Como resultado de ello se producen modificaciones en el marco en el que desarrollan su actividad.

A medida que los sistemas financieros se van desarrollando y tiene lugar el proceso de intervención de las economías de los distintos países, formando un mercado financiero internacional, surge la necesidad de estudiar con rigor la intensidad de la incidencia de determinados elementos del entorno sobre las principales magnitudes o indicadores de la situación financiera de las empresas.

El análisis económico-financiero centra fundamentalmente su atención en los movimientos que se producen en las estructuras económicas y financieras, por una parte, y por otro lado en el estudio de las variaciones que tienen lugar en los flujos financiero de la empresa.

En este trabajo se toman en consideración aquellas situaciones relacionadas con alternativas de inversión $<\mathrm{C}>$ en términos económicos y de proyectos de inversión, a los cuales se pueden asociar una serie de beneficios netos o egresos netos en dinero; estas cantidades de di- nero se pueden ubicar en el futuro, lo cual implica que las decisiones tienen un determinado nivel de incertidumbre. En algunos casos los resultados asociados a un determinado curso de acción son muy difíciles de evaluar en términos monetarios.

Algunas de las consideraciones difíciles de cuantificar son de tipo ético, moral, social, económico, político, estético, entre otros, y por el hecho de no ser cuantificables no deben ser despreciadas. De hecho, muchas decisiones que aparentemente desde el punto de vista económico $y$ financiero son aceptables, son rechazadas por los decisores, debido a factores de índole político-social.

Cuando el analista de proyectos solo tiene en cuenta los elementos de tipo económico y financiero cuantificables y muchas veces su recomendación no es atendida por la alta dirección en este caso, no debe pensarse que la evaluación fue incorrecta o que ella no se tuvo en cuenta. Lo que suele suceder es que la toma de decisión final involucra más elementos de juicio que no se encuentran en el análisis económico de alternativas.

\section{El análisis financiero}

Entre los elementos que tradicionalmente se utilizan para el análisis económico-financiero destaca por su interés el llamado métodos de los ratios. Se entiende por ratios un conciente entre elementos o masa patrimoniales que permite poner de manifiesto una particularidad de la situación económica o financiera. Se trata de expresar mediante una cifra la representación de algún aspecto de la actividad económica-financiera. 
Herramientas financieras del mercado venezolano como alternativas de inversión Atencio Cárdenas, Edith; González, Alejandro; González, Blanca y Katiuska Marín

Desde una perspectiva tradicional, los ratios ofrecen una gama de posibilidades para determinar tanto la situación de una empresa en un momento dado como la evolución de la misma a lo largo del tiempo, y también su posicionamiento frente a otras empresas de la concurrencia. Así pues, se pueden considerar cuatro tipos de ratios basados en:

a) análisis estático. Se considera un balance y una cuenta de resultados de los que se extraen los datos que informan sobre la situación de la empresa en un determinado momento.

b) estática comparativa. Se compara la situación de empresas en distintos momentos. No se estudia su trayectoria de manera continua. Sino la diferencia entre dos o más situaciones. Normalmente se utiliza para estudiar la evolución histórica de la empresa en relación a unos elementos predeterminados.

\subsection{Estudio secuencial de las modificaciones del ratio de endeudamiento}

El ratio de endeudamiento es un elemento que permite expresar de manera simplificada la estructura financiera de una empresa, a través del cociente de dos magnitudes agregadas del pasivo que representan las fuentes de financiación ajena y las de financiación propia.

Uno de los problemas mas importantes que se plantean en el análisis financiero viene dado por el establecimiento de la incidencia de las variaciones de la estructura financiera sobre el coste del capital y el valor de la empresa.

Existen estudios importantes que recogen trabajos elaborados por diversos autores, entre ellos Modigliani y Millar (2003), los cuales ponen de manifiesto con una formulación analítica y con ejemplos numéricos, lo que sucede con el coste del capital y con el valor de la empresa cuando varia su ratio de endeudamiento como consecuencia de aumentar o disminuir las deudas y disminuir o aumentar los capitales propios, bajo ciertas hipótesis restrictivas. Con este mecanismo se llegan a determinadas conclusiones, una de las más comúnmente repetidas dice que el coste de capital disminuya a medida que aumenta el ratio de endeudamiento. Evidentemente, al modificar las hipótesis de partida varían también las conclusiones, se pone de así de manifiesto una gama de situaciones identificadas que dan lugar a la elaboración de modelos que son conocidos, unos por el nombre de su autor o autores, otros por la principal hipótesis de partida.

Es evidente que los supuestos sobre los que se sustentan estos modelos no se hallan agotados con los trabajos publicados y que es posible la elaboración de nuevos esquemas con base en hipótesis distintas. Existe, pues, un camino abierto a nuevas investigaciones, que, proporcionan resultados interesantes.

\subsection{Relación entre la estructura financiera y el coste de capital}

La importancia de este tema justifica el hecho de que prácticamente todas las obras publicadas sobre gestión financiera hacen una referencia, mas o menos extensa, a la estructura financiera optima de la empresa. En este sentido, la polémica sobre la existencia misma de una estructura optima ha llenado miles de pagi- 
nas y dividido a los especialistas en posiciones mas o menos radicalizadas que se sitúan desde la afirmación rotunda de que es posible hallar relación entre las diferentes masas patrimoniales del pasivo que maximice los objetivos de la empresa, hasta la creencia a ultranza de que estos objetivos se pueden alcanzar con independencia de que se de una u otra estructura financiera.

Por lo tanto opiniones tan distintas solo se puedan justificar si se persiguen objetivos distintos y/o se parte de hipótesis diferentes, por ello, en una primera aproximación, se puede cuadricular el problema de la estructura financiera óptima según los diferentes objetivos de la empresa y escoger, para cada uno de ellos, la variada gama de supuestos en que se basan los distintos autores. En este sentido se puede suponer, por ejemplo, que el objetivo que se pretende alcanzar es la maximización de la utilidad de los accionistas y se desea ver que influencia ejerce los cambios de la estructura financiera sobre el coste del capital y sobre el valor que la empresa merece a los accionistas.

\subsection{Estrategias secuénciales para la captación de medios financieros}

Giuni et al. (2001) considera que uno de los aspectos básicos en la actividad financiera de las empresas se halla en sus procesos de captación de medios de pago. Estos pueden ser muy variados y de naturaleza diversa. Desde una perspectiva general se puede considerar que el empresario, para seguir desarrollando el proceso productivo precisa una determinada cantidad de medios monetarios para ir atendiendo las necesidades de tesorería a lo largo del tiempo.

Las empresas que se hallan inmersas en un sistema económico con expansión continua contempla, con frecuencia como sus flujos monetarios derivados de la facturación no siempre resultan suficientes.

Así, mientras una parte de estos son susceptibles de ser aportados por los mismos propietarios de la empresa y convertirse, así, en capitales propios y, otros es posible obtenerla por el excedente obtenido por la venta de los productos o servicios.

En este sentido, el contexto en el cual los hechos y fenómenos se suceden a velocidad vertiginosa, se plantea la necesidad de poner a disposición de las empresas un conjunto de técnicas que permiten a sus ejecutivos escoger aquellos productos financieros que mejor se adapten a sus necesidades.

Existen, en la literatura financiera, multitud de elementos que, incorporados a ciertos modelos de carácter determinístico o aleatorios, intentan dar una solución satisfactoria a la cuestión planteada. Sin embargo, creemos que un entorno eminentemente cambiante, es preciso utilizar otros tipos de instrumentos que permitan el tratamiento de los problemas financieros de la empresa en un contexto de incertidumbre.

\subsubsection{La Selección de productos fi-} nancieros

La captación de medios financieros por parte de las empresas plantea un problema de decisión como consecuencia de las plantea un problema de decisión como consecuencia de la variedad de productos financieros que los bancos y 
Herramientas financieras del mercado venezolano como alternativas de inversión Atencio Cárdenas, Edith; González, Alejandro; González, Blanca y Katiuska Marín

demás instituciones crediticias ponen a disposición de sus potenciales clientes.

Se observa, cada vez con mayor frecuencia, que aparecen en el mercado nuevo productos bajo formas muy diversas que, real o aparentemente, tienen características distintas. No se puede olvidar que la fuerte competitividad que caracteriza el universo financiero, obliga a los oferentes de medios de pago a un esfuerzo de diversificación y diferenciación de productos que les permitan, por una parte, cubrir el mas amplio abanico de demandantes y por otra, provocar una imperfección a través de la presentación de productos distintos con objeto de evadir las inexorables leyes del mercado perfecto.

Cuando se plantea la necesidad de recurrir a la financiación externa, los ejecutivos de las empresas se encuentran, pues, ante un número finito de opciones que ofrecen en el mercado, entre las cuales debe elegir aquella que mejor se adapte a sus propios requerimientos.

Lo anterior explica, porque para cada empresa, e incluso para cada situación concreta, existirá una distinta valoración de cada una de las características de los productos financieros. Así, en determinados casos, resulta muy importante la rapidez de la obtención de los medios financieros, en otros privará el plazo de devolución. En definitiva, el empresario estimará en cada circunstancia un orden de prelación con las características que configuran los productos.

En este contexto aparecen dos elementos fundamentales que conforman el problema plateado que son:

1. Diferenciación en las características de cada uno de los productos financieros que ofrecen.
2. Distinta estimación, por parte del demandante, de cada característica en relación con los demás, lo que proporciona un orden de preferencia.

Uno de los problemas fundamentales inherentes a la actividad financiera de la empresa viene determinando por la captación de recursos financieros que pueden ser de distinta procedencia, y cuya finalidad consiste en poder atender el normal funcionamiento de su propia actividad.

El objetivo de captar aquellos medios financieros, para la empresa, que comporten el menor coste posible, es una cuestión que ha sido ya tratada en diferentes trabajos. El punto de partida, que va a definir la aplicación de las estructuras, se asienta en el paralelismo existente entre la configuración de un grafo y la de una red de neuronas. De esta forma, asimilando ambos conceptos y aplicando los principios y propiedades de la teoría de grafos a las estructuras neuronales, se consigue establecer un esquema que permite escoger aquel o aquellos medios financieros mas adecuados a las necesidades sentidas por la empresa.

1.3.2. Criterios clásicos de valoración

Existe una especial unanimidad en la afirmación de que los intercambios económicos tienen lugar en áreas cada vez más amplias y que las relaciones financieras entre las empresas de diferentes países son cada vez mas estrechas. Asistimos a un proceso de interconexión e integración de los sistemas económicos en grandes bloques que, a su vez, mantienen intereses, a veces comunes, a veces contrapuestos, pero siempre interrelacionados. 
En este complejo mosaico de intereses aparecen, cada vez mas con mayor frecuencia, procesos de compra, absorción, concentración o fusión de empresas que exigen establecer una valuación que permita fijar un precio de compraventa aceptable por los eventuales adquiridos y vendedores de la misma.

Una de las características mas destacadas del problema de la valoración viene dada por el hecho de que difícilmente se puede obtener una estandarización en la fijación del precio, habida cuenta de que cada operación de compraventa de una empresa es diferente a las demás, y ello por motivos tan diversos como la singularidad de la empresa, sus estructuras económicas-financieras, su propia organización, los mercados en que intervienen, su entorno político, y un largo etcétera.

Por otra parte, la fijación de un precio que permita la transmisión de la titularidad de una empresa (o parte de una empresa) desde un grupo financiero a otro, no es el resultado puntual, la mayor parte de las veces, de un estudio técnico, sino consecuencia de laboriosas negociaciones en las que inician el dialogo partiendo de posiciones mas o menos alejadas. La misión del análisis financiero debe consistir en facilitar la misión a los negociadores, suministrando datos de partida suficiente fiables y a través de las técnicas disponibles, propiciar la convención hasta hacer posible el acuerdo.

\section{Decisiones financieras en la empresa}

La empresa como unidad básica de la economía, junto con los hogares o uni- dades familiares; es objeto de estudio de la microeconomía, pero todo el conjunto, reacciones y decisiones que se toman en su interior constituyen, de manera agregada, el objeto de estudio de la macroeconomía. Las finanzas de la empresa son una extensión operativa de la economía. En todo este proceso, se debe generar un superávit para que la economía crezca. Si en el proceso no se desarrollan estos excedentes, entonces la economía tenderá a desaparecer. Para que ello ocurra debe existir ahorro y una capacidad para que el dinero se reproduzca. El ahorro es el excedente de los hogares que pueden entregar a las empresas; la capacidad de reproducción del dinero es precisamente el papel que desempeñan los negocios de las empresas en su quehacer cotidiano y que producen, como resultado, el crecimiento económico.

Su propósito es generar riquezas que debe ser repartida en forma equitativa entre los diferentes miembros de la sociedad. Este reparto ocurre a través de varios mecanismos: una remuneración justa que permita una vida digna a los trabajadores, reparto adecuado de utilidades o dividendos a quienes han aportado el capital; pago de intereses razonables a quienes han suministrado fondos adicionales para que la empresa pueda operar, transferencias o redistribuciones que hace el Estado ,a través de los impuestos para subsidiar a los pobres, vía seguro social, organismos de cooperación social o prestación de servicios comunitarios entre otros.

Según autores como Modigliani y Millar (2003) fueron los primeros estudiosos de los métodos y aproximaciones para la toma de decisiones financieras, 
Herramientas financieras del mercado venezolano como alternativas de inversión Atencio Cárdenas, Edith; González, Alejandro; González, Blanca y Katiuska Marín

desarrollados en la década de los años 50. Ellos establecieron que los puntos críticos en el manejo financiero de una empresa eran tres.

1. Las decisiones de inversión.

2. La decisión sobre la estructura de capital.

3. La decisión de cómo repartir disidentes o política de dividendos.

Estos autores alegaron que el valor del mercado de una empresa era independiente de su estructura de capital y de la política de dividendos, de esta manera, el objetivo financiero de ella era identificar y emprender inversiones cuyos beneficios netos superaran sus costos netos. Los cuales están relacionados con la alternativa de inversión elegida que maximicen el valor presente neto (VPN).

En este particular y enfocado al sistema financiero de la empresa, particularmente el subsistema de inversión, se estudiaran todas y cada una de las herramientas financieras necesarias para Ilevar acabo de manera productivas las diferentes alternativas de inversión que nos presenta nuestro mercado financiero venezolano.

\section{Crecimiento e inversión financiera en la pequeña y mediana empresa venezolana}

La relación entre el crecimiento de la empresa y la política de financiamiento suele estar instalada en el imaginario colectivo de los administradores financieros y también en los directivos de las empresas. Es resultado de la perspectiva funcional y del enfoque externo.

La estructura de financiamiento es la combinación de las fuentes financieras de diversa naturaleza a las que recurre una empresa para financiar a sus activos tangibles e intangibles.

La posición jurídica de la empresa frente al proveedor del capital determina dos grandes categorias: el capital propio y el capital ajeno. El primero, el capital propio u operativo puede ser aumentado por la incorporación de nuevos socios o accionistas. El capital ajeno lo conforman las distintas obligaciones que pueden ser de corto y de largo plazo.

La formación de capital es una de las funciones de la empresa, es el resultado de las políticas de la empresa y de las decisiones de los directivos que se originan en el nivel de rentabilidad y en la tasa y el ritmo de capitalización. En el nivel macroeconómico el proceso de formación de capital tiene su origen en el proceso de ahorro, en el proceso financiero y en el proceso de inversión. En la empresa se necesita una mezcla de activos financieros y de activos tangibles financiados con una mezcla de fuentes tales como: el capital propio y las deudas sin incluir las otras fuentes.

Con los fondos generados por la inversión en activos financieros y tangibles que justifiquen tal inmovilización en términos de probable tasa de rentabilidad se debe tener en cuenta el riesgo empresario y el riesgo financiero.

Messuti (2001) plantea que el proceso de formación de capital y su ritmo de acumulación no asegura que la política de la empresa sea adecuada. El análisis clásico de contribución marginal nos dirá que, en todo momento, la firma deberá invertir mientras el fluir incremental de fondos generados por la inversión supere el costo de invertir para la firma. El análisis 
marginal es estático y no tomo en cuenta al tiempo como variable.

El proceso de acumulación considerado como un proceso dinámico es óptimo cuando cualquiera sea el estado y la decisión inicial, las decisiones restantes deben constituir una política óptima con respecto al estado resultante de la primera decisión. Por tanto las decisiones de inversión o de desinversión que es la expresión de las estrategias corporativas están vinculadas a las decisiones de financiación y de beneficios, lo que marcará el ritmo y la composición del proceso de formación de capital. Para las pequeñas y medianas empresas, en general, tienen las siguientes opciones estratégicas: crecen, se mantienen, ser achican, se venden o desinvierten en el sector actual; pero lo casi inevitable, se encuentra en la capacidad de sumir el riesgo.

Una forma de enfrentarlo parcialmente será la política de retención de beneficios pero no quiere decir que si la empresa pierde competitividad en el largo plazo deberá recurrir a diversas fuentes de financiamiento o cambiar la estructura del capital.

En nuestro caso por tratarse de pequeñas y medianas empresas y por el objetivo de éste artículo, el rubro que nos interesa es el capital propio u operativo y en especial los beneficios retenidos expresados contablemente de diferentes modos al que se le asigna el criterio del costo de oportunidad. Aunque también es conveniente considerar la combinación adecuada de pasivo y de capital propio u operativo que determine el menor costo promedio del capital de la empresa. Un aspecto importante dentro de la política de financiamiento es determinar la mezcla óptima de las distintas fuentes y el costo correspondiente; siendo un indicador importante el índice de endeudamiento.

La política de financiamiento en síntesis se refiere a la elección de las fuentes de financiamiento, o la estructura de financiamiento para financiar los activos de la empresa, función que es privativa de la dirección de la dirección y que con mayor precisión se puede afirmar que pertenece al nivel de gobierno de la empresa. En las pequeñas y medianas empresas no es tan fácil distinguir los niveles políticos, de gobierno y gerencial. La política de financiamiento demuestra una actitud frente al riesgo de la dirección ante diferentes alternativas de financiación, algunas veces acotadas por la política monetaria del gobierno de turno y por sus efectos en el costo de las diferentes fuentes.

En éste aspecto existen diferentes enfoques, unos afirman que existe una estructura óptima de financiamiento y otros afirman que no. Es necesario considerar que cada actividad o sector tiene una estructura de financiamiento específica porque la composición de los activos también es diferente. El capital propio y el capital ajeno son dos factores variables y sustituibles entre sí, el capital propio no se mantiene fijo, pero en el caso que sucediera, éste hecho, hay que aumentar el endeudamiento que no haría viable la empresa.

La formación de capital es tarea típica de la empresa moderna, las pequeñas y medianas empresas cumplen un rol fundamental en este sentido, ya que para ellas en la actualidad es de suma importancia el saber seleccionar las posibles alternativas de inversión y tener claro el significado del capitalismo financiero, en el cual se confunden los grupos económi- 
Herramientas financieras del mercado venezolano como alternativas de inversión Atencio Cárdenas, Edith; González, Alejandro; González, Blanca y Katiuska Marín

cos, los grupos de empresas y los grupos financieros. Por lo que se ha llegado a afirmar que, el objetivo de la empresa no es solo proveer bienes y servicios a una comunidad o a la sociedad en general sino que su éxito y posicionamiento en el mercado, estará en función des la autoexpansión de su capital.

\subsection{Instrumentos tradicionales de inversión}

Actualmente a corto plazo los mercados financieros son aleatorios y a largo plazo, y en vista de la variedad de activos diversificados, pueden realizar inversiones que le brinden retornos positivos acordes con el riesgo que seleccione. Los activos financieros se clasifican usualmente en instrumentos de renta fija y renta variable. Además, pueden clasificarse de acuerdo con el desempeño correlacionado de todos los instrumentos de una misma clase. La expresión correlacionada se refiere a que los retornos a corto plazo de los instrumentos de una clase tienden a reaccionar en la misma dirección ante eventos que cambian su valor.

\subsubsection{Instrumentos de renta fija}

Entre las clases de activos de renta fija se distinguen los instrumentos transaccionales, los de riesgo soberano en mercados locales e internacionales, y la llamada renta fija empresarial. A esta clase de activos se puede acceder mediante instrumentos financieros tales como: cuentas de ahorro, depósitos a plazo, letras del tesoro, bonos de la deuda pública nacional (DPN), vebonos, instrumentos emitidos por el gobierno en mercados internacionales, europapeles comerciales, euronotas a mediano plazo, eurobonos y fondos mutuales de renta fija.

No es recomendable invertir en instrumentos empresariales o soberanos que hayan caído en algún tipo de atraso en el pago de sus acreencias y se hayan visto obligados a seguir un proceso de reestructuración. Los inversionistas tienen muy mala memoria y lo pagan caro. Únicamente así se puede explicar que muchos emisores-tanto países como empresas- hayan entrado en cese de pagos, pérdida total o parcial de los intereses o el capital, de forma reiterada. Muchos inversionistas no miden bien la capacidad de deterioro, no están bien asesorados o tienen mala memoria al invertir en algunos instrumentos soberanos. Por ejemplo Brasil cayó en cese de pagos en 1937 y 1983 y Argentina en 1989 y 2001.

3.1.2. Instrumentos de renta variable

Entre los activos de renta variable se distinguen las acciones en el mercado venezolano, mientras que en los mercados desarrollados de Estados Unidos y Europa y en los llamados mercados emergentes, países en diversas etapas de desarrollo que ofrecen oportunidades atractivas de inversión. A estos activos se puede acceder mediante instrumentos financieros tales como acciones, fondos cotizados y fondos mutuales de renta variable. No invierta en un instrumento particular de renta variable, a menos que lo utilice para configurar una canasta diversificada de acciones.

\subsubsection{Instrumentos transacciona-} les de renta fija

En ésta clase de instrumentos se encuentran las cuentas de ahorro y los depósitos a plazo. En teoría, no existe 
riesgo al invertir en estos instrumentos, al menos por el monto garantizado por el fondo de garantías de depósitos y protección bancaria (FOGADE). Sin embargo, hay diferentes entre tasas y plazos, si el monto invertido supera los límites de la garantía, entonces haga un análisis de cada institución financiera; si no tiene los conocimientos, el tiempo o las habilidades para realizar algún tipo de análisis, entonces diversifique sus ahorros en varias instituciones.

El desempeño financiero de estos instrumentos depende de las tasas de interés del mercado y de lo que las economías de escala y la productividad de algunas instituciones bancarias permitan ofrecer a sus clientes en forma de tasas pasivas.

\subsection{Renta de riesgo soberano en mercados locales}

Según Gil (2004) Entre estos instrumentos se encuentran las letras del tesoro, los DPN y los vebonos. Las letras del tesoro son instrumentos clásicos de tesorería a corto plazo. Las emisiones de deuda pública nacional tiene un plazo mayor; pero, al ser recompradas por el estado, algunas emisiones carecen de un mercado con suficiente liquides. Los vebonos son instrumentos de más largo plazo, utilizados principalmente por el estado para pagar obligaciones contractuales a trabajadores, que se negocian en la bolsa de valores de caracas (BVC).

Estos instrumentos no tienen, en teoría, riesgo a vencimiento, más allá del riesgo crediticio del Estado. Si el horizonte de inversión es menor que el vencimiento del papel, estará sujeto al riesgo de las expectativas de los operadores profesionales de éste mercado. Estas expectativas son generadas por complejas intuiciones acerca de las tasas de interés a diferentes plazos, la liquidez existente en el mercado, las necesidades financiamiento del Estado y las expectativas de los demás participantes, entre otros factores.

Las principales ventajas de estos instrumentos son su liquidez, la garantía del Estado y un rendimiento generalmente superior al que ofrecen los instrumentos transaccionales, están al alcance de cualquier inversionista con mucho o escaso patrimonio, necesitando para ello una cuenta de corretaje a la que asignara la titularidad de la custodia de la inversión, la cual es electrónica y bien organizada. Esas cuentas de corretaje se pueden obtener en casas de bolsas, sociedades de corretaje o bancos.

Una desventaja de estos instrumentos es que los montos de ésta inversión son ligeramente superiores a los requeridos por algunos instrumentos transaccionales. Además, si necesita liquidez en un momento dado antes del vencimiento pude ocurrir que el valor este afectado por circunstancias particulares del mercado; pero la encontrara siempre, al menos en caso de las letras del tesoro. Su desempeño financiero principalmente de las tasas de interés existentes en el mercado.

\subsection{Renta fija de riesgo soberano en mercados internacionales}

Esta clase de instrumentos consiste en las emisiones del estado en los mercados internacionales. Este mercado opera en dólares y en euros, con algunas emisiones en otras monedas internacionales. 
Herramientas financieras del mercado venezolano como alternativas de inversión Atencio Cárdenas, Edith; González, Alejandro; González, Blanca y Katiuska Marín

En esta clase de activos no existe vencimiento, más allá del riesgo crediticio del Estado medido como su disposición y capacidad para cumplir sus obligaciones financieras, nunca ha dejado de cumplir las obligaciones financieras contraídas en el extranjero. Prácticamente, todas las emisiones son clasificadas según su riesgo, y de esa clasificación depende el rendimiento que recibe el inversionista. Si su horizonte es menor que el vencimiento del papel, estará sujeto al riesgo derivado de las expectativas de los operadores profesionales de este mercado. Tales expectativas son generadas por las especulaciones de los operadores acerca de la disposición y la capacidad de pago del país.

Este es un mercado para profesionales y especuladores. Se convierte en una excelente alternativa de inversión, cuando se logra construir una cartera de instrumentos diversificados. Adicionalmente, estos instrumentos disponen de diferentes estructuras financieras que les permite adaptarse a diferentes grados de tolerancia al riego. Las emisiones principales cuentan con gran liquidez. Su principal desventaja es la volatilidad-variaciones de precios- de los mercados en los cuales se transan. Además, los eventos que afectan la calidad crediticia de cada Estado generalmente se magnifican.

\subsection{Renta fija empresarial}

Esta categoría incluye papeles comerciales, emisiones de bonos y titularizaciones que son papeles comerciales de emisiones a corto plazo y las titularizaciones tienen generalmente un plazo mayor.
Todas las emisiones sobre las que se realiza oferta pública en Venezuela están reguladas por la Comisión Nacional de Valores. Esta supervisión garantiza estándares mínimos, tanto de calidad de información suministrada al inversionista como de cumplimiento de lo establecido en la Ley de Mercado de Capitales y las normas respectivas. Todas las emisiones sujetas a oferta pública tienen que estar calificadas, con respecto a su riesgo, por más de una empresa calificadora debidamente autorizada, al momento de la emisión.

Entre las ventajas de estos instrumentos están la posibilidad de obtener una relación riesgo-retorno y un plazo que en algunos momentos no este disponible en el mercado bancario. La principal desventaja es la liquidez, que generalmente es nula y, cuando es muy baja, se elevan los costos asociados. Su desempeño financiero depende principalmente de la tasas de intereses existentes en el mercado, en el momento de la emisión, y de la capacidad y la disposición de pago de las empresas.

\subsection{Acciones en el mercado venezolano}

Las acciones son instrumentos emitidos por las empresas que otorgan derechos residuales (una vez cumplidas las obligaciones de las empresas) sobre su patrimonio. Tiene un riesgo considerable, especialmente si se invierte en una o muy pocas acciones a corto plazo. El riesgo fundamenta se deriva de los eventos del mercado y la actividad generadora de flujo de caja de cada empresa. 
El mercado venezolano es pequeño, de liquidez limitada (excepto para algunas acciones de las empresas) y con pocos operadores cada vez mas profesionales. Es una buena alternativa, solamente si se invierte a largo plazo en un portafolio diversificado, que ofrezca un rendimiento superior al de otras opciones. Además, el mercado local ha incorporado en los últimos años diferentes mecanismos que permiten mayor transparencia y supervisión adecuada. Entre sus desventajas se encuentran la escasa liquidez de muchas acciones y falta de número de emisiones que permitan al inversionista variar su cesta de opciones en diferentes sectores y empresas.

El desempeño de las acciones depende, a corto plazo, de las expectativas de los participantes acerca de los eventos de un mercado que es aleatorio. A largo plazo, los mercados se ajustan para que el inversionista obtenga una tasa acorde con el riesgo en que incurre.

\section{Principios básicos para el logro de decisiones asertivas en las inversiones.}

\subsection{Componentes de la estrategia de inversión}

Este es el punto de partida para el diseño de una estrategia de inversión, el cual consiste en: identificar la etapa en la cual se encuentra la empresa en su ciclo productivo; determinar sus componentes a corto, mediano y largo plazo, además considerar cualquier ahorro previo o participación en programas de ahorro. También es necesario analizar las características de la empresa,como es su nivel de productividad, rentabilidad y competitividad dentro del mercado en el que se desenvuelve, esta información es indispensable para establecer junto con el gerente o los asesores financieros con los que cuenta la empresa, los objetivos de inversión que, a su vez, determinan y son determinados por su estructura financiera, requeridos a lo largo de su ciclo de vida productiva y la combinación de activos apropiados parar poder cumplir tales objetivos.

Para Giugni et al. (2001) la mezcla de activos se refiere a la combinación de instrumentos de renta variable (acciones $\mathrm{y}$ fondos mutuales de renta variable), renta fija (bonos, papeles comerciales, certificados de depósitos y fondos mutuales de renta fija, entre otros) e inversiones alternativas (por ejemplo, bienes inmuebles) que una empresa puede ir agregando a sus activos, de acuerdo con su estrategia de inversión. Como bien postula la teoría financiera, los instrumentos en los que se percibe mayor riesgo suelen ofrecer mayores rendimientos, por lo que su importancia será mayor en las estrategias de crecimiento patrimonial de más largo plazo. Esto se debe a que estas inversiones pueden producir rendimientos negativos en algunos años, lo cuales se deberían compensar con los buenos rendimientos de los mejores años. Esta apuesta tiene probabilidad de éxito a medida que se alargue el plazo en el cual se realizan las inversiones.

El esfuerzo de ahorro depende, entre otros factores, de la capacidad acumulativa que tenga la empresa, este esfuerzo debe realizarse anualmente $y$ debe ser incrementado cuando el objetivo de reacumulación patrimonial del año 
Herramientas financieras del mercado venezolano como alternativas de inversión Atencio Cárdenas, Edith; González, Alejandro; González, Blanca y Katiuska Marín

previo no se haya alcanzado (como resultado de, por ejemplo, una baja de las cotizaciones del mercado accionario). Si bien la obtención de la información necesaria para diseñar una estrategia de inversión es responsabilidad del interesado, que éste la comparta con el asesor, quien deberá recomendar un monto anual y de ahorro y proponer una combinación de activos para la empresa.

\subsection{Riesgo de las inversiones}

Según Gil (2004). Entre estos instrumentos se encuentran las letras del tesoro, los DPN y los vebonos. Las letras del tesoro son instrumentos clásicos de tesorería a corto plazo. Las emisiones de deuda pública nacional tiene un plazo mayor; pero, al ser recompradas por el estado, algunas emisiones carecen de un mercado con suficiente liquidez. Los vebonos por el contrario son instrumentos de más largo plazo, utilizados principalmente por el estado para pagar obligaciones contractuales a trabajadores de la administración pública y, se negocian en la bolsa de valores de Caracas (BVC).

Estos instrumentos no tienen, en teoría, riesgo a vencimiento, más allá del riesgo crediticio del Estado, si el horizonte de inversión es menor que el vencimiento del papel, estará sujeto al riesgo de las expectativas de los operadores profesionales de éste mercado. Estas expectativas son generadas por completas intuiciones acerca de las tasas de interés a diferentes plazos, la liquidez existe en el mercado, sujetas a las necesidades de financiamiento del Estado y a las expectativas de los demás participantes, entre otros factores.
Las principales ventajas de estos instrumentos son su liquidez, la garantía del Estado y un rendimiento generalmente superior al que ofrecen los instrumentos trasaccionales, están al alcance de cualquier inversionista con mucho o escaso patrimonio, necesitando para ello una cuenta de corretaje a la que asigna la titularidad de la custodia de la inversión, la cual es electrónica y bien organizada. Esas cuentas de corretaje se pueden obtener en casas de bolsas, sociedades de corretaje o bancos.

Una desventaja de estos instrumentos es que los montos de ésta inversión son ligeramente superiores a los requeridos por algunos instrumentos transaccionales. Además, si necesita liquidez en un momento dado antes del vencimiento puede ocurrir que el valor este afectado por circunstancias particulares del mercado; pero la encontrara siempre, al menos en caso de las letras del tesoro. Su desempeño financiero principalmente dependerá de las tasas de interés existente en el mercado.

Todo inversionista debe comprender los riesgos que entrañan las diferentes opciones de inversión existentes. Debido a que las empresas tienen aversión al riesgo, sin embargo, estarán dispuestas a invertir en instrumentos de mayor riesgo en la medida en que les ofrezcan una remuneración adicional por incorporarse a su cartera.

El riesgo no es más que un cálculo de la probabilidad de que los pagos prometidos por una inversión no se materialicen, tal como se espera en el momento de realizarla. Los resultados de una inversión pueden ser sustancialmente diferentes de lo esperado, sea por exceso o por 
defecto. Una inversión se considera de mayor riesgo en la medida en que su rendimiento esperado fluctúe más ampliamente a lo largo del tiempo, con el consiguiente aumento de la probabilidad de experimentar pérdidas importantes cuando la inversión pierde valor.

Hay un tipo de riesgo que ninguna persona u empresa debería asumir; el riesgo que resulta de catástrofes tales como: incendios, inundaciones, muerte y terremotos, entre otras. Este tipo de riesgos debe ser contrarestado con pólizas de seguros.

\subsection{Efectos de la inflación}

Uno de los mayores retos de las empresas, si se tiene un horizonte de inversión a mediano o largo plazo, consiste en acumular recursos e incrementar su poder adquisitivo a lo largo del tiempo, por estar invertidos en bienes o instrumentos financieros cuyo valor se rezaga con respecto a la tasa de inflación. En ese caso la empresa tendrá que asumir un deterioro importante de localidad productiva de sus inversiones. Cuando se consideran los efectos de la inflación se comprende la magnitud de la apuesta que se hace al seleccionar una determinada combinación de activos. La mezcla de activos determina no sólo la volatilidad o fluctuación de los rendimientos que producen los instrumentos incluidos en la cartera de inversión, sino también su exposición a la inflación.

Todo aquel inversionista que haya colocado una proporción importante de su dinero en depósitos a plazo en bolívares, durante las dos últimas décadas, ha sufrido descapitalización, Ello se debió a que los recursos fueron invertidos a tasas de interés que, en promedio, resultaron reales negativas; es decir, que estuvieron por debajo de la tasa de inflación observada. La inversión en acciones de empresas venezolanas habría ofrecido un mayor crecimiento patrimonial, incluso positivo en términos reales, a costa de asumir un mayor riesgo. Lo mismo habría ocurrido en el caso de invertir en acciones de empresas norteamericanas, aunque el desempeño fue superior.

\subsection{Diversificación de la cartera de inversiones}

Uno de los postulados más importantes de la teoría de carteras se refiere a que, en vista de su supuesta aversión al riesgo, los inversionistas deben diversificar sus carteras entre los distintos instrumentos existentes. Al internacionalizar una cartera de inversiones los beneficios de la diversificación se pueden potenciar, pues se logra desvincular una porción de la inversión de las fluctuaciones de una economía particular. Al diversificar apropiadamente una cartera, tanto por sectores como por países incluidos se compensan, lo cual suaviza el desempeño del retorno global del inversionista.

Hoy no se necesita grandes cantidades de dinero para construir una cartera diversificada internacionalmente, basada en adquirir participaciones en fondos mutuales que inviertan internacionalmente o invertir en acciones de empresas de distintos países al abrir una cuenta de inversión en internet. En todo caso, el costo de diversificar internacionalmente una cartera (por comisiones y otros gastos) es mucho menor hoy que hace apenas una o dos décadas atrás. Entre los Tipos de Inversión tenemos: 
Herramientas financieras del mercado venezolano como alternativas de inversión Atencio Cárdenas, Edith; González, Alejandro; González, Blanca y Katiuska Marín

\subsubsection{Inversión Productiva}

La producción en el sistema económico, es uno de los indicadores más importantes, por consiguiente necesita de la inversión, para que esta pueda ser efectiva y, la podemos definir como el gasto en bienes de capital, para generar otros bienes, que por consecuencia de su venta, darán una ganancia; puede ser bruta, cuando se toma el valor de la cantidad invertida y o neta cuando a la inversión bruta le restamos la depreciación de los factores productivos. La productividad y eficiencia en la producción, es siempre uno de los Principales objetivos de toda empresa, por esa razón, buscará las alternativas con mayor rentabilidad.

La inversión representa el gasto en que incurren las familias, las empresas y el sector publico, en bienes que al transcurrir un tiempo, ya sea largo o corto, traerán como consecuencia un beneficio. La inversión es válida, sólo para la compra de bienes de capital, financieros o intermediarios, porque ellos después de atravesar un determinado proceso, serán bienes de consumo final, es una variable, que por lo general, se toma en cuenta en base al futuro, debido a que el inversionista no constituirá una empresa, para vender en el mercado sólo su primera producción y luego cerrar la empresa, el verdadero propósito, es una inversión constante en la empresa, para generar un beneficio por un largo periodo.

En este sentido la inversión consta de muchas clasificaciones, ya que puede darse en variadas condiciones tales como: la procedencia del capital, en el tipo de bienes en que se invierte, entre otros, a partir de allí puede ser: a largo plazo, a corto plazo, nacional, ex- tranjera, pública, privada o, aquellas en que incurren los empresarios, para comenzar un nuevo negocio, para aumentar los insumos, mano de obra, mejorar las maquinarias y todos aquellos factores, que de una u otra forma influyen en el volumen de producción, con el objetivo de aumentarla o mejorarla, para obtener mayores y mejores beneficios.

\subsubsection{Inversión Financiera}

La inversión financiera consiste en la adquisición de activos financieros con ánimo de inversión, no tiene una función económica directa, no tiene una finalidad productiva o de prestación de servicio, sino que es una inversión en activos reales, representados en bienes que sirven para producir otros bienes, pero la inversión financiera cumple sin embargo una función económica de gran importancia, posibilitando la transferencia de fondos (esquema financiación-inversión) y de riesgos (sirva como ejemplo que al comprar acciones de una empresa estamos asumiendo proporcionalmente parte del riesgo de la compañía).

Los distintos productos financieros emitidos cumplen una función relacionada con la transferencia de fondos o de riesgos. La emisión y contratación de acciones en el mercado secundario (en otras palabras de segunda mano) no contribuye de forma directa al crecimiento del PIB o, a la formación bruta de capital, pero si facilita la financiación del crecimiento empresarial, con lo que esto supone para el PIB o la formación bruta de Capital.

\subsubsection{La inversión inmobiliaria}

Borga (2004) Opina que, las empresas al realizar sus inversiones espe- 
ran un rendimiento futuro, además suele representar una parte muy importante de sus portafolios de activos. La inversión en bienes raíces tiene ventajas y desventajas que cada inversionista pondera de manera diferentes.

Entre las ventajas de la inversión inmobiliaria se destacan las siguientes: los inmuebles permiten un apalancamiento financiero mayor que los instrumentos financieros, debido a la posibilidad de adquirirlos mediante créditos hipotecarios; es posible diversificar geográficamente el portafolio de un inversionista al invertir en fondos mutuales de bienes raíces, como son las compañías de inversión inmobiliarias (REIT), por las siglas de real estate (investiment trusts); las propiedades ofrecen una buena protección con respecto a la inflación y los inversionistas pueden realizar mejoras que aumentan el valor de mercado de los inmuebles.

Entre las desventajas es justo mencionar que, las transacciones inmobiliarias suelen tener gran impacto en el patrimonio de los inversionistas, el costo de obtener información en el sector inmobiliario es significativo, los costos de transacciones son relativamente altos (comisiones de compra y venta, y costos de registros e impuestos) y los gastos de mantenimiento y administración pueden ser elevados, los bienes tienen ubicación fija y están expuestos a riesgos tales como el deterioro de la urbanización en la cual estén ubicados.

\subsection{Empleo inteligente los ingresos extraordinarios}

Siempre que haya dinero extra producto de utilidades o dividendos, el inver- sionista disciplinado debe analizar qué hacer para utilizarlo de un modo consistente con la estrategia de inversión trazada. Por ejemplo, debe dar prioridad al pago de deudas a corto plazo, que tienden a ser fuentes de financiamiento más costosas. Además, debe crear un fondo de reserva, el cual debe constituirse aunque no tengamos ingresos extraordinarios y antes de invertir recursos a largo plazo. La recepción de ingresos extraordinarios es una magnifica oportunidad para mejorar la calidad de vida de la empresa, púes permite eliminar compromisos financieros relativamente onerosos.

\subsection{Efecto de factores económicos sobre las decisiones de inversión}

Según Baca (2005) un manejo deficiente de la planificación tributaria puede resultar muy costoso a mediano y largo plazo, en la medida en que los bolívares adicionales (de las ganancias o los intereses) restados por los impuestos no pueden ser reinvertidos. En este punto es clave la labor del asesor financiero, al recomendar las inversiones más favorables desde el punto de vista impositivo. En Estados Unidos, por ejemplo, las ganancias de capital son pechadas a una tasa fija independiente del ingreso, mientras que los dividendos son pechados como ingreso corriente. Dado que en ese país la estructura de impuesto sobre la renta es progresiva, todas aquellas empresas o personas que devengan mayores ingresos deberían realizar un mayor pago de impuesto, no sólo en dólares sino también como porcentaje del ingreso percibido. Por ello, a las personas y empresas de altos ingresos o ganancias se suele re- 
Herramientas financieras del mercado venezolano como alternativas de inversión Atencio Cárdenas, Edith; González, Alejandro; González, Blanca y Katiuska Marín

comendar invertir en acciones de empresas que pagan dividendos relativamente bajos, los cuales suelen experimentar mayores ganancias de capital.

\section{Instrumentos de Inversión recomendadas a las PYMES venezolanas}

Una política financiera es aquella que establece los lineamientos relacionados con la adquisición, uso y distribución entre los propietarios, del capital de la empresa. En virtud de esta definición, las políticas financieras se establecen en:

- campo del financiamiento

- campo de la inversión

- campo de los dividendos

Las políticas de financiamiento son aquellas que establecen las pautas en relación con la provisión de capital por las diferentes fuentes de financiamiento, y las mismas se refieren a la capacidad de endeudamiento, las posibilidades de financiamiento a través de capital propio, las formas de suplir las necesidades de capital a corto plazo, entre otros, que tiene una empresa en un momento determinado y en cuyo establecimiento privan factores tales como: costo de capital, compromisos adquiridos con los suplidores de capital y destino de los fondos obtenidos.

En las políticas de inversión están incluidas aquellas relacionadas con la utilización del capital en adquisición en activos fijos y activos circulantes. Las políticas de inversión en activos fijos cubren aspectos tales como áreas de inversión, tasa mínima de rendimiento, niveles de riesgo, criterios de selección entre alternativas de inversión, entre otros; en cambio, las que regulan la inversión en activos circulantes se refieren a tamaños de inventarios, límites de capital asignado a cuentas por cobrar, índice de liquidez, etc.

Según Vélez (1998), las políticas de dividendos se refieren al establecimiento de los lineamientos fundamentales en la repartición de los beneficios obtenidos por la venta del bien producido o servicio prestado. Es de hacer notar que se distribuyen dividendos en aquellas compañías constituidas por acciones. Cuando esta situación no se presenta, estas políticas definen el destino que se le da a los beneficios percibidos una vez que han sido cancelados los compromisos de deudas adquiridos. En su fijación intervienen factores tales como el deseo de beneficio de los accionistas, requerimientos de capital de inversión y aspectos legales.

Gil (2004) menciona que las políticas financieras se interrelacionan con todas las políticas de una empresa, como por ejemplo, una política de ampliación de las instalaciones productivas tiene que ver con la posibilidad de penetrar nuevos mercados; una limitación del crédito en las ventas afecta directamente el servicio que se da al consumidor. Las políticas financieras también se afectan entre sí, por ejemplo, la cantidad de capital disponible para la inversión depende de las políticas de repartición de dividendos; las políticas de inversión en proyectos rentables influyen en las políticas para la búsqueda de nuevas fuentes de financiamiento. En consecuencia, su estudio y análisis es de gran importancia por las aplicaciones que tienen dentro del establecimiento y funcionamiento de un sistema empresarial. 
Es necesario, una vez más, resaltar que una política financiera es un lineamiento que sirve de guía y, dentro de la cual deben estar enmarcadas todas las actividades que se ejecutan en la empresa en el campo financiero; no obstante, estas actividades son el resultado de un proceso de toma de decisiones acorde con las líneas definidas, por lo que en forma general la literatura se refiere concretamente a:

- Decisiones de financiamiento

- Decisiones de inversión

- Decisiones de dividendos

En la Figura 1 se ilustran las políticas y decisiones financieras de una empresa.

\section{Figura 1}

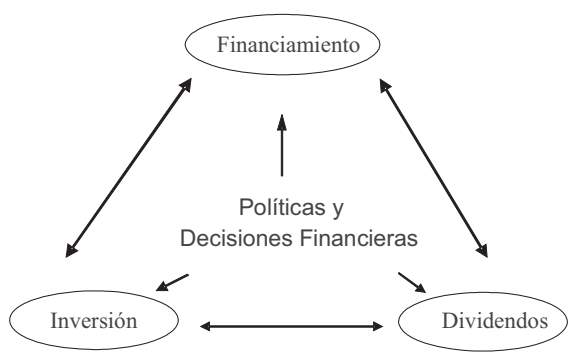

Elaboración propia (2008).

\section{Decisiones de financiamiento}

Vélez (1998) comenta que las decisiones de financiamiento son aquellas que permiten establecer la mejor estructura de capital para la empresa. En otras palabras, ello significa decidir la cantidad y la calidad del aporte de capital proveniente de cada una de las diferentes fuentes de financiamiento.
La Figura 2 señala las principales fuentes de capital que utiliza una empresa, las cuales básicamente se clasifican: de capital propio, si el mismo es aportado por los dueños o generados como producto de su gestión, y de capital de deuda si proviene de fuentes externas en calidad de préstamos.

\section{Figura 2}

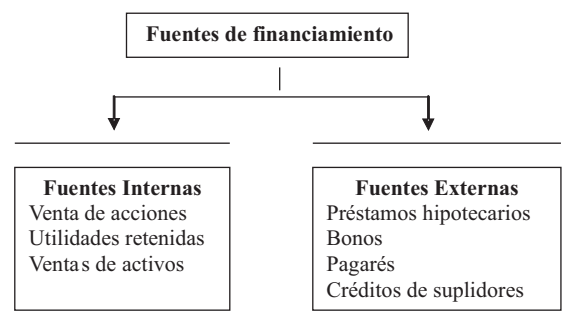

Elaboración Propia (2008).

Principales fuentes de financiamiento que utiliza la empresa

Decisiones de Inversión

Son aquellas que permiten determinar las formas de utilizar el capital en la adquisición de los activos de la empresa. Las decisiones de inversión se clasifican en:

a) Decisiones de Inversión a largo plazo, que se refieren a la adquisición de activos fijos. por ejemplo, la edificación de un galpón industrial, la compra de una maquinaria de producción. entre otros activos.

b) Decisiones de inversión a corto plazo, que se llevan a cabo con la finalidad de adquirir activos cuya duración dentro de la empresa abarca periodos menores a un año; tal es el caso de los activos circulantes. Por ejemplo, la inversión en inventarios de materia prima y productos terminados, 
Herramientas financieras del mercado venezolano como alternativas de inversión Atencio Cárdenas, Edith; González, Alejandro; González, Blanca y Katiuska Marín

en cuentas por cobrar, en dinero en efectivo, entre otros. Estas inversiones generalmente se producen como consecuencia de las primeras, ya que no existen activos circulantes si previamente no existen activos fijos.

En resumen, cualquier desembolso de dinero, utilizado con la finalidad de adquirir activos fijos y circulantes, que se espera produzca beneficios en periodos mayores que un año, recibe el nombre de inversión de capital. Desde este punto de vista, una oportunidad de inversión o proyecto se puede definir como la actividad técnica, que puesta en marcha, permite el logro de beneficios futuros de inversiones de capital.

Ejemplo:

La ampliación de una línea de producción representa para una empresa la realización de un proyecto, ya que se supone la ejecución de una serie de actividades de orden técnico que conducen al aumento de la producción y, por ende, con expectativas de que se aumenten los beneficios futuro. Ahora bien, llevar a cabo este proyecto puede significar. Inversiones de largo plazo

- La construcción de un nuevo galpón.

- La adquisición e instalación de equipos adicionales.

Inversiones de corto plazo

- El aumento de los inventarios

Todo lo anterior constituye inversiones de capital.

\section{Decisiones de Dividendos}

Las decisiones de dividendos se refieren a la determinación de la cantidad de beneficios o dividendos a repartir entre accionistas. El monto de los dividendos no sólo depende de los deseos de beneficios de los propietarios sino también de los objetivos de la empresa y de sus necesidades de capital de inversión.

En consecuencia, las decisiones de dividendos varían periódicamente en función de los elementos anteriormente mencionados. Por ejemplo, en los períodos donde se presenten más posibilidades de inversión, la tendencia será a repartir menor cantidad de beneficios que, en aquellos en los cuales las oportunidades de invertir sean más escasas.

En el caso especial de las organizaciones que no están constituidas por acciones, ya sea por que tengan un solo propietario o porque sean de una naturaleza diferente, estas decisiones se identifican con aquellas relacionadas con la repartición de utilidades a sus socios.

En tal sentido Vélez (1998) Opina que, tal como ocurre en el caso de las políticas financieras, estas tres decisiones no se producen nunca en forma aislada, sino que existe, por el contrario, una intima relación entre ellas, ya que se afectan mutuamente. Así, una decisión de financiamiento puede afectar las decisiones de dividendos, en el sentido de que si por ejemplo, el financiamiento a utilizar se basa en utilidades retenidas, ello afectará los dividendos repartidos y viceversa, si los accionistas exigen una mayor cantidad de dividendos habrá menos capital disponible para financiar los proyectos y muy posiblemente las decisiones de inversión cambien.

Cabe destacar que dentro de las decisiones financieras sobresalen en importancia las decisiones de inversión, por cuanto, son las que permiten el crecimiento del 
capital a través de la obtención de beneficios, lo cual trae como consecuencia el desarrollo de las organizaciones.

\section{Conclusiones}

En la vida de las organizaciones, siempre se presentan situaciones por resolver. Las formas de solucionarlas son variadas y por lo general, con recursos escasos. Al presentarse diversas alternativas de solución, es razonable pensar en seleccionar la mejor de ellas, la función de un gerente es tomar decisiones siempre con restricciones o bajo criterios de escasez; pero en la práctica hay exceso o cantidades ilimitadas de recursos, no hay dificultades en la elección. Sin embargo aún en la abundancia, habrá que escoger un curso de acción. Debe tenerse claro la importancia de maximizar los excedentes que producen las empresas. En la medida en que esto ocurra, la sociedad como un todo se beneficiará ya que habrá más recursos para repartir.

El Estado juega un papel muy importante; para que una economía de libre mercado funcione bien, es necesario contar con un Estado fuerte que administre justicia entre los asociados. No se trata de un Estado totalitario, ni un Estado de enormes dimensiones, sino de un árbitro que permita lograr corregir fallas del mercado. Las decisiones deben tomar en cuenta cómo se logra el bienestar de todos los actores del proceso económico. Esto implica, que aún las decisiones que aparentemente son evidentes desde el punto de vista estrictamente financiero, deben consultar aspectos tales como efectos sobre el medio ambiente, efectos sociales sobre los empleados y clientes, efectos económicos sobre pro- veedores y sobre quienes suministran servicios a la empresa.

La educación financiera de la persona o gerente que lleva las riendas de una empresa desempeñan un papel fundamental dentro y fuera ella, en la medida en que el inversionista conozca los instrumentos disponibles y los mercados, se encontrará en mejor capacidad para estructurar la estrategia de inversión con su asesor financiero y sentirse más cómodo con los instrumentos financieros que la integran, pues entiende sus características de rendimiento y riesgo percibido.

Por lo tanto las cifras financieras no son suficientes; hay que tener un adecuado conocimiento del negocio, de las tendencias del mercado, de los cambios tecnológicos y de los posibles movimientos de la competencia. Todo esto se puede asociar a la intuición. Debe observarse sin embargo, que la intuición es una flor silvestre; tiene fundamentos no solo vivénciales y empíricos, sino también de formación académica.

\section{Referencias Bibliográficas}

Baca Urbina, Gabriel (2005). Evaluación de Proyectos. Cuarta Edición. McGrawHill. México.

Borga, Anna (2004). Inmuebles: una inversión atractiva en Venezuela? Debates IESA. Vol IX. No. 3.

Gil Lafuente, Anna (2004). Nuevas Estrategias paralelas al análisis financiero de la empresa. Editorial Ariel. Buenos Aires-Argentina.

Giugni de Alvarado, Luz; Ettedgui de Betancourt, Corina, et al. (2001). Evaluación de Proyectos de Inversión. Segunda Edición. Clemente Editores C.A. Universidad de Carabobo. Valencia-Venezuela. 
Herramientas financieras del mercado venezolano como alternativas de inversión Atencio Cárdenas, Edith; González, Alejandro; González, Blanca y Katiuska Marín

Messuti, Domingo (2001). Finanzas de la Empresa: Un enfoque a nivel ejecutivo.

Modigliani y Millar (2003). La Financiación de las Empresas. Cuadernos de Economía, V. XXII, No. 39. Bogota. pág. 85 y 86.
Vélez Pareja, Ignacio (1998). Decisiones de Inversión: una aproximación al análisis de alternativas. Primera Edición. Pontificia Universidad Javeriana. Bogotá-Colombia. 